\title{
Die COVID-19 Pandemie als Herausforderung und Chance für Register in der Versorgungsforschung: Erfahrungen aus Lean European Open Survey on SARS-CoV-2 Infected Patients (LEOSS)
}

The COVID-19 Pandemic as an Opportunity and Challenge for Registries in Health Services Research: Lessons Learned from the Lean European Open Survey on SARS-CoV-2 Infected Patients (LEOSS)

\author{
Autoren \\ Lisa Pilgram1 ${ }^{\mathbb{D}}$, Maximilian Schons², Carolin E.M. Jakob², 3, Annika Y. Claßen², 3, Bernd Franke2, 3, Lene Tscharntke², \\ Nick Schulze2, 3, Sandra Fuhrmann², Gabriel Sauer², Susana M. Nunes de Miranda², Fabian Prasser ${ }^{4,5}$, Melanie Stecher ${ }^{2,3}$, \\ Jörg J. Vehreschild1, 2, 3
}

Institute

1 Hämatologie, Onkologie, Hämostaseologie, Rheumatologie, Infektiologie, Universitätsklinikum Frankfurt Zentrum der Inneren Medizin, Frankfurt am Main, Deutschland

2 Onkologie, Hämatologie, Klinische Infektiologie, Klinische Immunologie, Hämostaseologie, Internistische Intensivmedizin, Uniklinik Köln Klinik I für Innere Medizin, Köln, Deutschland

3 Standort Köln-Bonn, Deutsches Zentrum für Infektionsforschung (DZIF), Köln, Deutschland

4 Charité Universitätsmedizin Berlin, Berlin Institute of Health, Berlin, Deutschland

5 Corporate member of Freie Universität Berlin and Humboldt Universität zu Berlin, Charité Universitätsmedizin Berlin, Berlin, Deutschland

\section{Schlüsselwörter}

COVID-19, Pandemie, Register, Versorgungsforschung, Netzwerk, Open Science

Key words

COVID-19, Pandemic preparedness, Registry, Health services research, Network, Integrative research

\section{Bibliografie}

Gesundheitswesen 2021; 83 (Suppl. 1): S45-S53

DOI 10.1055/a-1655-8705

ISSN 0949-7013

(C) 2021. Thieme. All rights reserved.

Georg Thieme Verlag, Rüdigerstraße 14,

70469 Stuttgart, Germany

\author{
Korrespondenzadresse \\ Dr. Lisa Pilgram \\ Hämatologie, Onkologie, Hämostaseologie, \\ Rheumatologie, Infektiologie \\ Universitätsklinikum Frankfurt Zentrum der Inneren Medizin \\ Theodor-Stern-Kai 7 \\ 60590 Frankfurt am Main \\ Deutschland \\ lisa.pilgram@leoss.net
}

\section{ZUSAMMENFASSUNG}

Ziel der Studie Aus der durch das Severe Acute Respiratory Syndrome Coronavirus 2 (SARS-CoV-2) bedingten CoronavirusKrankheit-2019 (COVID-19) haben sich Chancen und Herausforderungen für den Aufbau von Registern in der Versorgungsforschung ergeben. Diese sollen exemplarisch am aktuell größten sektorenübergreifenden Register mit einem detaillierten klinischen Datensatz zu mit SARS-CoV-2 infizierten Patient:innen in Deutschland, der Lean European Open Survey on SARS-CoV-2 Infected Patients (LEOSS), aufgezeigt werden. Methodik Ziele von LEOSS waren es, ein kollaboratives und integratives Register zur Erfassung von anonymen Daten aus der Versorgung zu schaffen und die Daten der Wissenschaft im Sinne eines Open Science Ansatzes rasch bereitzustellen. Alleiniges Einschlusskriterium war der virologische Nachweis von SARS-CoV-2. Schlüsselstrategien waren die Reallokation der vorhandenen personellen und technischen Ressourcen, die frühe und direkte Einbeziehung von Vertreter:innen des Datenschutzes und der Ethikkommissionen sowie die Entscheidung zu einem iterativen und agilen Entwicklungs- und Anpassungsprozess.

Ergebnisse Getragen von den zahlreichen kollaborierenden Institutionen konnte ein transsektorales und internationales Netzwerk mit aktuell 133 aktiv rekrutierenden Standorten und 7227 dokumentierten Fällen aufgebaut werden (Stand 
18.03.2021, ein Jahr seit Rekrutierungsstart von LEOSS). Die Nutzung der Daten wurde über auf der Projektwebseite verfügbare Werkzeuge zur Datenexploration, wie auch über die teilautomatisierte Bereitstellung von Datensätzen verschiedenen Umfangs, innerhalb kurzer Zeit ermöglicht. Es wurden 97 Anträge zur Datennutzung aus 27 Themengebieten begutachtet. Im Peer-Review-Verfahren wurden 9 Arbeiten in internationalen Fachzeitschriften veröffentlicht.

Schlussfolgerung Mit LEOSS konnte in kürzester Zeit ein System zur Erfassung klinischer Verlaufsdaten zu COVID-19 in Deutschland etabliert werden. Auch wenn in anderen Projekten für spezifische Fragestellungen weitaus größere Datenbestände durch direkten Zugriff auf Quellsysteme analysiert werden konnten, wurde durch den einheitlich gepflegten und technisch geprüften Dokumentationsstandard mit vielen fachspezifischen Details ein sehr großer Datensatz mit wertvollen Alleinstellungsmerkmalen geschaffen. Aus den Erfahrungen von LEOSS können Implikationen für die zukünftige Gestaltung von Registern und eine rasche Reaktion auf Pandemien abgeleitet werden.

\section{ABSTRACT}

Objective The Coronavirus Disease-2019(COVID-19) pandemic has brought opportunities and challenges, especially for health services research based on routine data. In this article we will demonstrate this by presenting lessons learned from establishing the currently largest registry in Germany providing a detailed clinical dataset on Severe Acute Respiratory Syndrome Coronavirus 2 (SARS-CoV-2) infected patients: the Lean European Open Survey on SARS-CoV-2 Infected Patients (LEOSS).
Methods LEOSS is based on a collaborative and integrative research approach with anonymous recruitment and collection of routine data and the early provision of data in an open science context. The only requirement for inclusion was a SARSCoV-2 infection confirmed by virological diagnosis. Crucial strategies to successfully realize the project included the dynamic reallocation of available staff and technical resources, an early and direct involvement of data protection experts and the ethics committee as well as the decision for an iterative and dynamic process of improvement and further development.

Results Thanks to the commitment of numerous institutions, a transsectoral and transnational network of currently 133 actively recruiting sites with 7,227 documented cases could be established (status: 18.03.2021). Tools for data exploration on the project website, as well as the partially automated provision of datasets according to use cases with varying requirements, enabled us to utilize the data collected within a short period of time. Data use and access processes were carried out for 97 proposals assigned to 27 different research areas. So far, nine articles have been published in peer-reviewed international journals.

Conclusion As a collaborative effort of the whole network, LEOSS developed into a large collection of clinical data on COVID-19 in Germany. Even though in other international projects, much larger data sets could be analysed to investigate specific research questions through direct access to source systems, the uniformly maintained and technically verified documentation standard with many discipline-specific details resulted in a large valuable data set with unique characteristics. The lessons learned while establishing LEOSS during the current pandemic have already created important implications for the design of future registries and for pandemic preparedness and response.

\section{Einleitung}

Ausgehend von Wuhan in der Provinz Hubai in China hat sich Ende Dezember 2019 das Severe Acute Respiratory Syndrome Coronavirus 2 (SARS-CoV-2) global verbreitet. Ende Januar 2020 wurde mit der Feststellung einer gesundheitlichen Notlage internationaler Tragweite durch die WHO [1] und den ersten Fällen in Deutschland [2] die dringende Handlungsnotwendigkeit deutlich. Wie bereits zu Beginn prognostiziert, entwickelte sich die Pandemie der Coronavirus-Krankheit-2019 (COVID-19) zu einer weltweiten Bedrohung mit über 122 Mio. Infizierten und über 2,5 Mio. Todesfällen (Stand 22.03.2021) [3] und stellt seit Pandemiebeginn Gesellschaften, Gesundheitssysteme und Volkswirtschaften vor große Herausforderungen.

Die COVID-19 Pandemie ist die sechste gesundheitliche Notlage internationaler Tragweite, die seit Inkrafttreten der überarbeiteten Internationalen Gesundheitsvorschriften (IGV) 2005 deklariert wurde. Damit wurde die globale Forschungsgemeinschaft klar aufgefordert, schnellstmöglich eine Evidenzbasis für Entscheidungsträger:innen zur Verfügung zu stellen [1].
In der klinischen Forschung, im Bereich Public Health, wie auch in der Versorgungsforschung, leisten Register einen wesentlichen Beitrag zur Bereitstellung von Evidenz aus der alltäglichen Gesundheitsversorgung und können damit komplementär zu klinischen Studien wirken. Im Kontext der Planung für und des Managements von Pandemien (Pandemic Preparedness and Response) sind Daten aus der Versorgung und die Zeit- und Kosteneffizienz von Registern zur Sammlung verlässlicher klinischer Daten besonders relevant [4]. Dies zeigte sich bereits in vorangegangenen Ausbruchsgeschehen wie 2003 bei SARS [5] oder 2009/10 bei Influenza A [6]. Um dies zu erreichen, sind Vernetzung, Schnelligkeit, Ressourcenschonung, zielgenaue Erhebung und Skalierbarkeit bereits in die Konzeptionierung einzubeziehen. In der Realisierung fordert die Erforschung eines unbekannten Erregers ein hohes Maß an Flexibilität, um beispielsweise bei SARS-CoV-2 sich ändernde Falldefinitionen einzubeziehen [7] oder erste Erkenntnisse wie den frühen Hinweis auf Gerinnungsstörungen gezielt zu adressieren [8]. Die zeitkritische Lieferung einer Evidenzbasis unter Einbezug von Qualitätsanforderungen und -prüfungsverfahren [9], Leitlinien für Gute Epidemiologische Praxis (GEP) [10] und zur Nachhaltigkeit [11] stellt eine 
besondere Herausforderung dar, bietet aber auch eine Chance für Register in der Pandemie.

Für Register mit einer offenen Fragestellung sind Repräsentativität und eine ausreichende Fallzahl von besonderer Bedeutung. Wenn beides erreicht wird, können wichtige Rückschlüsse für die Gesamtheit der Patient:innen getroffen und die Betrachtung seltener Effektgrößen wie bspw. Komedikation und Komorbidität ermöglicht werden. Vor diesem Hintergrund bestand zunächst die Hoffnung, dass sich das internationale und von der WHO unterstützte Register des International Severe and Acute Respiratory and Emerging Infection Consortium (ISARIC, https://isaric.org/) auch in Deutschland durchsetzen könnte, was jedoch, wahrscheinlich aufgrund datenschutzrechtlicher Limitationen, fehlender deutscher Studiendokumente und eines starken Fokus auf intensivmedizinische Maßnahmen, nicht gelang.

Im vorliegenden Beitrag soll nun der Aufbau des Registers im Kontext der COVID-19 Pandemie beschrieben und unter den Gesichtspunkten der zeitkritischen Konzeption und Umsetzung, sowie der Anforderungen an Qualität und Nachhaltigkeit evaluiert und im internationalen Zusammenhang diskutiert werden.

\section{Methodik}

Auf Initiative der Deutschen Gesellschaft für Infektiologie (DGI) mit der Emerging Infections Task Force (EITaF) der European Society of Clinical Microbiology and Infectious Diseases (ESCMID) in Zusammenarbeit mit dem Deutschen Zentrum für Infektionsforschung (DZIF) sollte ein transnationales und -sektorales Netzwerk unter dem Titel Lean European Open Survey on SARS-CoV-2 Infected Patients (LEOSS) entstehen.

Im Hinblick auf die unvorhersehbare Entwicklung der COVID-19 Pandemie wie auch der Dynamik verschiedener Forschungsinitiativen wurden die Konzeptionierung und Umsetzung als kontinuierlicher Prozess verstanden. Damit wurden wesentliche Voraussetzungen bereits vor Rekrutierungsbeginn geschaffen, die kontinuierliche Weiterentwicklung erfolgte jedoch auch während der Projektrealisierung.

\section{Rekrutierung}

Durch die anonyme Erfassung von Fällen aller Schweregrade sowie den transsektoralen und internationalen Ansatz sollten Verzerrungen in der Rekrutierung vermieden werden. Dies ist hinsichtlich des hohen Anteils an schwer erkrankten (nicht-einwilligungsfähigen) Patient:innen eine notwendige Voraussetzung, um die Epidemiologie und den klinischen Verlauf von COVID-19 umfassend abzubilden.

Alleiniges Einschlusskriterium von LEOSS war und ist der positive labormedizinische Nachweis von SARS-CoV-2, sodass LEOSS das Spektrum von rein ambulant behandelten Fällen bis hin zur intensivmedizinischen Komplexbehandlung nicht einwilligungsfähiger Patient:innen abdeckt. Während erste Netzwerkpartner:innen sich bereits aktiv in die Konzeptionierung einbrachten, wurden parallel Gespräche mit internationalen medizinischen Fachgesellschaften sowie Gesundheitsforschungszentren gesucht, um deren fachliche und politische Unterstützung einzuholen.

\section{Verwendete Technologien}

Aufgrund der raschen Verfügbarkeit (existierende Lizenz der Arbeitsgruppe), positiver Vorerfahrungen und der intrinsischen Vernetzungsfunktionen wurde die auf EFS Survey (Questback AG, Köln) basierende ClinicalSurveys.net Plattform als Electronic Data Capture System verwendet. Die Prozessierung, Aufbereitung und Prüfung der Daten, wie auch die Prüfung der Anonymisierung erfolgte in R, die Anonymisierung selbst in Java. Der Datentransfer an Antragsteller:innen erfolgte in verschlüsselten Archiven über die DRACOON-Plattform (DRACOON GmbH, Regensburg) der Uniklinik Köln mit 2-Faktor-Authentifizierung.

\section{Flexibilität und Skalierbarkeit in der Datenerhebung}

Bei initial noch unklarem Krankheits- und Pandemieverlauf war Flexibilität bei der Datenerhebung unabdingbar. Hierfür wurden im Protokoll flexible Rekrutierungszahlen und -zeiträume, wie auch umfassende Studienziele festgelegt. Zudem wurden Fachgruppen und weitere Interessenvertreter:innen, bspw. der Deutschen Zentren der Gesundheitsforschung, gezielt angesprochen, die Erfassung zu prüfen und ggf. Modulvorschläge zu machen.

Die umfangreiche Dokumentation wurde durch JavaScript gestützte Filter- und Ausblendbedingungen erleichtert.

\section{Anforderungen an den Datenschutz}

Aufgrund der bekannten Unsicherheiten in Bezug auf die Verarbeitung anonymisierter Daten [12] wurde in Absprache mit dem Datenschutzbeauftragten der Universität zu Köln ein zweistufiges Anonymisierungskonzept entworfen.

Im ersten Schritt wurde eine Erfassungssystematik gewählt, die eine Reidentifizierung bereits mit hoher Sicherheit ausschließt: auf personenidentifizierende Daten wurde vollständig verzichtet, metrischer Werte wurden kategorisiert, die exakte Zeitachse durch Krankheitsphasen ersetzt, einzelne Datenelemente über diese Phasen aggregiert und phänotypische Merkmale wurden weitgehend nicht erfasst.

Zusätzlich wurde ein zweiter Anonymisierungsschritt in einer zertifizierten sicheren Netzwerkumgebung durchgeführt, wo mit externer Beratung eine Risikoeinstufung bezüglich Verknüpfbarkeit (linkability), Replizierbarkeit (replicability) und Verfügbarkeit (availability) [13] für mögliche Deanonymisierungsversuche für alle Datenpunkte erfolgte und über Aggregation und Zensierung von Datenelementen weitere Maßnahmen zur Verhinderung einer hypothetischen Reidentifizierung ergriffen wurden [14].

\section{Zugang und Nutzung der Daten innerhalb kurzer Zeit}

Mit der Registrierung im Deutschen Register Klinischer Studien (DRKS) wie auch der Übertragung der Datenformulare auf das Portal für Medizinische Datenmodelle (MDM) der Universität Münster $[15,16]$ wurden im Projektverlauf auffindbare und zugängliche Metadaten geschaffen.

Für das Teilen der Daten wurden unterschiedliche Bereitstellungen vorgesehen: (a) der vollständige lokale Datensatz der jeweiligen Standorte, (b) eine eingeschränkte Datenbasis aus 16 Variablen (Public Use File, PUF), öffentlich verfügbar auf der Webseite und (c) eine breite Datenbasis (Scientific Use File, SUF) zur Beantwortung von komplexen Forschungsfragen. Der Vorschlag, den SUF interessierten Wissenschaftler:innen unter dem Open Science 
Gedanken auch ohne Nutzungsverfahren zur Verfügung zu stellen, wurde in den Entscheidungsorganen mehrheitlich kritisch gesehen. Zentrale Argumente gegen ein freies Teilen der Daten waren (i) die mangelnde Qualitätssicherung der durchgeführten wissenschaftlichen Untersuchungen, mit Gefahr für Dopplungen, Widersprüche und methodisch schwachen Analysen und (ii) die Sorge um eine unzureichende Honorierung der freien Zuarbeit durch das Netzwerk in der Konzeption und in der Datensammlung. Als Kompromisslösung wurde ein schlankes Antragsverfahren mit kurzen Umlaufzeiten und Verzicht auf ein Quorum zur Antragsannahme entwickelt.

\section{Qualitätssicherung}

Qualitätssichernde Prozesse erfolgten in der Datenerhebung unter Berücksichtigung der anonymen Patient:innenrekrutierung über die zentrale Überprüfung der Qualifizierung der Dokumentierenden und auf Stufe der Dateneingabe. Letzteres wurde durch automatisierte Vollständigkeits- und Plausibilitätsprüfungen, hinterlegte klinische Definitionen und Eingabehilfen umgesetzt.

Voraussetzungen zur Datenauswertung beinhalteten im Protokoll festgehaltene personelle wie auch methodische Anforderungen. Nur Auswertungen durch qualifiziertes Personal unter Nutzung adäquater Methoden und Software waren zulässig.

\section{Steuerungsgremien}

Für einen fairen und integrativen Ansatz wurden drei Steuerungsgremien definiert: Zentrale Entscheidungskompetenzen für Studienprozesse wie auch Datennutzungsanträge wurden für die Standorte mit der höchsten Rekrutierungsleistung nach einer transparenten Metrik (Board of Investigators, Bol) definiert. Ein wissenschaftlicher Fachbeirat (Global Scientific Council, GSC) mit Einbindung in das Datennutzungsverfahren wurde aus den beteiligten Fachgesellschaften und Forschungseinrichtungen zusammengesetzt. Größere Entscheidungen und umfassende Berichte werden in der Vollversammlung (General Assembly) diskutiert.

\section{Ergebnisse}

\section{Initiierung und Rekrutierung}

Nach intensiven Diskussionen über die Notwendigkeit eines weiteren Registers neben der ISARIC-Initiative und unter dem Eindruck einer rasch ansteigenden ersten Welle wurde am 09.03.2020 der Entschluss gefasst, das LEOSS-Register zu etablieren ( $\triangleright$ Abb. 1).

Angesichts der Dringlichkeit entwickelte ein Kernteam von sechs erfahrenen Mitarbeiter:innen aus den Bereichen Epidemiologie, Medizin und Medizininformatik innerhalb von 48 Stunden einen ersten Entwurf von Fragebogen, Studienprotokoll und Homepage. Die Entwürfe von Studienprotokoll und Anonymisierungskonzept wurden dann in direkter Absprache mit der Ethikkommission des Universitätsklinikums Köln und dem Datenschutz der Universität zu Köln weiterentwickelt. Am 16.03.2020 erfolgte die Einreichung parallel bei den Ethikkommissionen an den Universitätsklinika Frankfurt und Köln, die in Dringlichkeitssitzungen jeweils am 16.03.2020 und 19.03.2020 ein positives Votum erteilten. Am 18.03.2020 wurde der erste Fall am Universitätsklinikum Frankfurt dokumentiert.

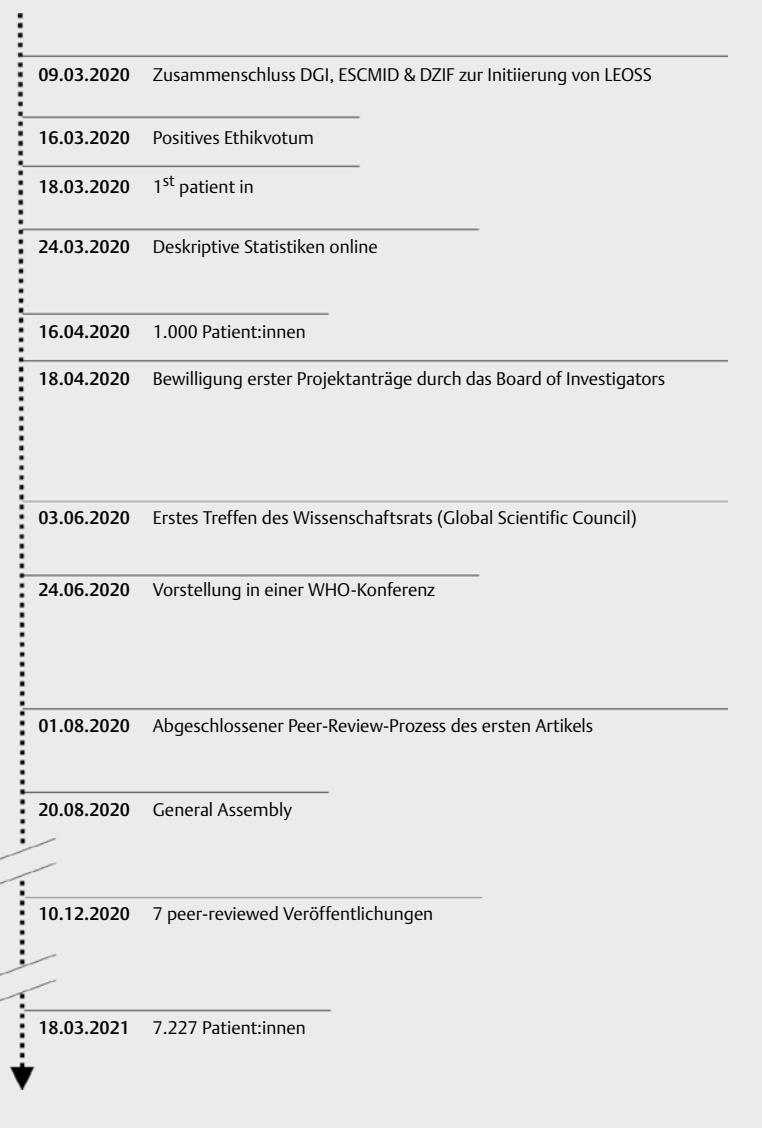

Abb. 1 Zeitliche Abfolge relevanter Ereignisse von Initiierung bis 1 Jahr nach Rekrutierungsstart in LEOSS.

Basierend auf den initialen Ethikvoten folgte die flächendeckende Einreichung an universitären Kliniken und Landesärztekammern. Ende März 2020 lagen bereits 37 positive Voten vor. Der rasche Ausbau der Rekrutierung mit Einbeziehung weiterer Standorte erfolgte initial maßgeblich durch die Unterstützung der DGI, der ESCMID und des DZIF, und durch das Zurückgreifen auf bereits etablierte Netzwerke der Arbeitsgruppe aus Projekten wie der Translationalen Plattform HIV (TP-HIV, https://tp-hiv.de/) oder der multizentrischen, internationalen PILGRIM-Studie. Im weiteren Verlauf wurden zunehmend über die weiteren Deutschen Zentren der Gesundheitsforschung (DZG) und zahlreiche medizinische Fachgesellschaften Standorte angebunden. Die proaktive Bewerbung des Registers erfolgte parallel über die Nutzung dementsprechender Verteiler, frühe Pressemeldungen [17-22] und soziale Medien wie Twitter. Der gemeinsame Aufruf führte letztlich zu einer Anzahl von aktuell 133 aktiv rekrutierenden Standorten vorwiegend in Deutschland, jedoch auch über die Grenzen hinaus (Stand 18.03.2021, siehe $>$ Abb. 2).

Die Dokumentationsrate pro Woche stieg initial exponentiell an ( Abb. 3). Wesentliche Meilensteine in der Rekrutierung stellten 1000 Patient:innen nach weniger als einem Monat, 3000 im Juli 2020 und eine Fallzahl von 7.227 (Median = 15, unteres Quartil = 3, oberes Quartil=62; Stand 18.03.2021) ein Jahr nach dem ersten Patient:inneneinschluss dar. Hierbei sind 4,6\% (330/7227) der Re- 


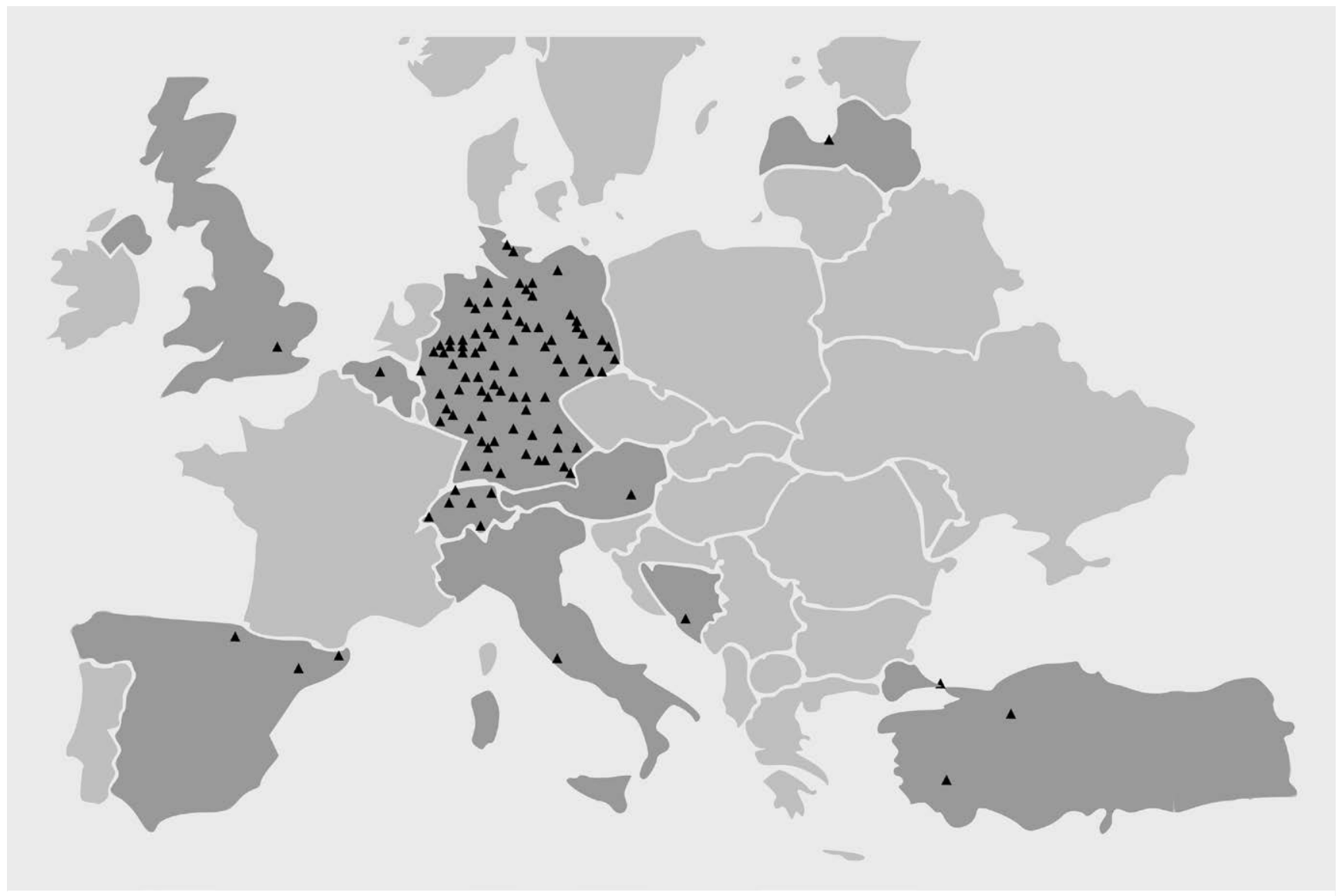

Abb. 2 Aktive Standorte. Ein Standort gilt dann als aktiv, wenn er einen Beitrag von mindestens einem vollständigen Fall nach Registrierung geleistet hat. Stand 18.03.2021.

krutierungen außerhalb von Deutschland und 44,5\% (3214/7227) außerhalb des universitären Sektors zu verorten.

\section{Entwicklung des Datensatzes}

Der Datensatz wurde unter Einbezug lokaler Erfahrungen, externer Anfragen und Zuarbeiten sowie auf Basis neuer Erkenntnisse iterativ an aktuelle Fragestellungen und Versorgungsrealitäten angepasst. Substanzielle Anpassungen wurden in den Gremien der Studie verabschiedet. Hierdurch wuchs die Anzahl an Variablen im Projektverlauf von 400 auf 2241 an (Stand 18.03.2021). Insgesamt wurden an 78 Tagen Erweiterungsschritte aktiv gesetzt, die zunächst in einer Testumgebung implementiert, anschließend in die Aktivumgebung kopiert und über ein Änderungsverzeichnis offengelegt wurden. Die im Projektverlauf etablierte Testumgebung und Handlungsanweisung vermieden erfolgreich Fehler in der Implementierung trotz hoher Änderungsdichte.

\section{Datennutzung}

Innerhalb eines Monats nach Rekrutierungsbeginn wurde das PUF öffentlich verfügbar gemacht. Zeitgleich waren die ersten deskriptiven Analysen der Gesamtkohorte auf der Webseite einsehbar. Webbasierte Applikationen (Dashboard, Jupyter Notebook) zur Exploration der Daten wurden teilweise durch freiwillige Externe (PROCON IT GmbH, Garching) gestaltet, wodurch die Nutzung des PUF wesentlich vereinfacht wurde.
Seit Beginn wurden weit über 100 Anfragen zur Nutzung der LEOSS-Daten gestellt, nach formaler Vorprüfung wurden insgesamt 97 Anträge aus 26 Themengebieten durch die Gremien begutachtet und bis auf wenige Ausnahmen innerhalb von zwei Wochen positiv votiert. Ein enger Austausch zwischen den Gruppen wurde gefördert und Projekte im Verlauf fusioniert. Die häufigsten Themenbereiche waren Methodologie/Techniken der künstlichen Intelligenz (12 Projekte), Herz-Kreislaufsystem (8 Projekte) und Neurologie (7 Projekte). Nach Eingang der detaillierten Aufstellung der benötigten Daten dauerte es zwischen wenigen Tagen und 6 Wochen, bis der angepasste Datensatz nach Durchlaufen aller beschriebenen Prüfungen übermittelt werden konnte. Durch die enge Anbindung an eine für LEOSS aufgebaute Transferstelle wurde eine Unterstützung der Arbeitsgruppen aufgebaut, sodass mittlerweile acht weitere fachspezifische Forschungsarbeiten aus den Bereichen Kardiologie, Nephrologie, Neurologie, Onkologie, Transplantationsmedizin und zur Methodologie in internationalen Fachzeitschriften publiziert werden konnten [14, 23-30].

\section{Herausforderungen}

Einhergehend mit der Geschwindigkeit der Anbindung war die zentrale Koordination der zahlreichen Standorte eine Herausforderung. Zusätzlich führte der zweite Anonymisierungsschritt mit maßgeschneiderter Anpassung des Datensatzes für die Transfer- 


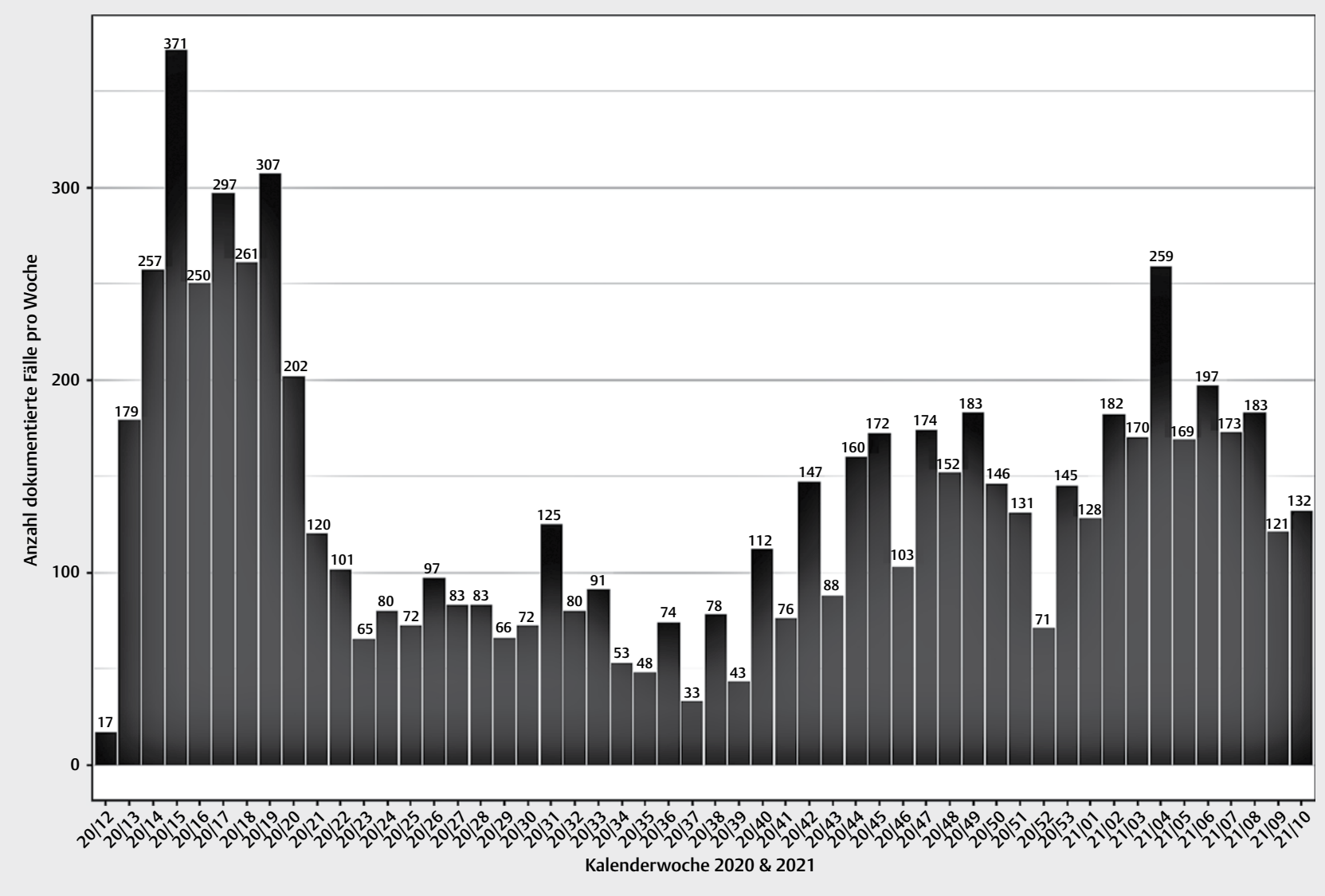

Abb. 3 Dokumentierte Fälle pro Woche. Die Dokumentationsrate pro Woche ist für ein Jahr ab Rekrutierungsstart in LEOSS dargestellt. Die Anzeige der Kalenderwoche setzt sich zusammen aus den letzten Ziffern des Jahres und dem Monat und beziffert den Zeitpunkt der Falldokumentation, nicht der Diagnose. Gesamt- $\mathrm{N}=7179$.

stelle zu einem erheblichen Zeitaufwand von bis zu 80 Stunden pro Datensatz.

Die hohe klinische Auslastung und stark zunehmende Anzahl verschiedener Register im April und Mai ließ in den Zentren vermehrt den Wunsch nach einer zumindest teilweise elektronischen Datenübermittlung aufkommen. Diese konnte aufgrund unzureichender finanzieller Mittel und der für einen direkten Import in die Datenbank notwendigen umfassenden Validierung solcher Schnittstellen nicht umgesetzt werden.

\section{Diskussion}

Nach unserer Kenntnis zählt LEOSS nach ISARIC bis heute zu den größten nach einheitlichem Schema erfassten, interdisziplinären, transsektoralen und transnationalen Datensammlungen zu COVID19 und leistet neben eigenen Analysen relevante Beiträge zu europäischen und internationalen Datensammlungen. Wichtige Beispiele für Projekte, zu denen LEOSS beigetragen hat, umfassen Registry of patients with COVID-19 including cardiovascular risk and complications (CAPACITY, https://capacity-covid.eu/), Connecting European cohorts to increase common and effective response to SARS-CoV-2 pandemic (ORCHESTRA, https://orchestra-cohort.eu/) und die Global COVID-19 Clinical Data Platform der WHO. Das Fehlen rasch verfügbarer Ressourcen konnte durch die offene Projekt- struktur und die faire Verwendung der erhobenen Daten kompensiert werden, sodass eine hohe Motivation zur Teilnahme bestand.

Der naheliegendste internationale Vergleich zu LEOSS ist die ISARIC Datenerhebung, die mit einer Gesamtanzahl von mehr als 100000 Fällen die größte uns bekannte zentralisierte Datensammlung zu COVID-19 mit offener Fragestellung darstellt. ISARIC bietet ein Tool zur Erfassung der lokalen Daten der Studienstandorte an, mit der Option, Daten für gemeinsame Auswertungen zur Verfügung zu stellen. Hierbei bestanden keine Verpflichtungen bzgl. der Erfassungstiefe: Während am 14.04.2021 Daten zu Komorbiditäten für 127750 Fälle vorlagen, war z. B. die Gabe von Betablockern nur für 1339 Patient:innen dokumentiert [31].

Als Alternative zu prospektiven Registern besteht international zunehmend die Möglichkeit der direkten Nachnutzung elektronisch verfügbarer Gesundheitsdaten, wodurch teilweise große Fallmengen und Kontrollgruppen betrachtet werden konnten. In Großbritannien konnte bspw. für über 2 Mio. Bürger:innen der Gebrauch von Medikamenten aus klinischen Routinedaten mit Details aus Sterbedaten verknüpft werden [32], in Südkorea wurden anonymisierte Krankenkassendaten für wissenschaftliche Forschung geteilt, die Informationen über 200000 SARS-CoV-2 Infizierte beinhalteten und auch die Betrachtung spezifischer Komorbiditäten ermöglichten [33]. Bei entsprechenden Dateninfrastrukturen und etablierten regulatorischen und datenschutzrechtlichen Prozessen erlaubt 


\section{LEOSS Lessons Learned}

\section{- Datenschutz}

$\checkmark$ Einbezug von Ethikkommission und Datenschützer:innen bereits in die Konzeptionierung

$\checkmark$ Keine Erhebung direkt-identifizierbarer Daten

$\checkmark$ Zusätzliche Maßnahmen zur Reduktion des Re-Identifizierungsrisikos

- Proaktive Bewerbung und Sichtbarkeit

$\checkmark$ Paralleler Aufruf über Pressemitteilungen, Social-Media-Kanäle und Verteiler kooperierender medizinischer Fachgesellschaften

$\checkmark$ Kurzfristige Implementierung und kontinuierliche Aktualisierung von Projektwebseite und Newsletter

$\checkmark$ Öffentliche Bereitstellung anonymisierter Datensätze, Analysetools und deskriptiver Statistiken

- Niedrigschwelliger Zugang für teilnehmende Standorte

$\checkmark$ Flächendeckende Einreichung an Ethikkommissionen von Ärztekammern und Universitätsklinika

$\checkmark$ Webbasiertes eCRF

$\checkmark$ Initial wöchentliche Unterstützungsangebote (z.B. im Rahmen von offenen Telefonkonferenzen)

- Integrativer Ansatz

$\checkmark$ Kollaboration mit und Unterstützung von zahlreichen (inter)nationalen medizinischen Fachgesellschaften, Forschungsinstitutionen und -initiativen

$\checkmark$ Iterative Entwicklung des Datensatzes durch die Einbindung von Fachexpert:innen

$\checkmark$ Vernetzung verschiedener Interessensgruppen

- Datenzugang und -nutzung

$\checkmark$ Abruf lokaler Datensätze ohne Antragsverfahren

$\checkmark$ Open Governance unter Einbezug von Standortvertreter:innen und Wissenschaftler:innen kooperierender Fachgesellschaften und Institutionen

$\checkmark$ Schlanke Entscheidungsprozesse ohne Quorum und unter engen zeitlichen Vorgaben

$\checkmark$ Berücksichtigung rekrutierender Standorte bei Forschungsprojekten (Autor:innenregulation)

- Transparenz

$\checkmark$ Verfügbarkeit von Metadaten

$\checkmark$ eCRF-Testzugänge

$\checkmark$ Änderungsverzeichnis

$\checkmark$ Frei zugängliche Datennutzungsordnung

- Abb. 4 LEOSS Lessons Learned. Mögliche Implikationen im Aufbau zukünftiger Register insbesondere aber nicht ausschließlich im Kontext von Pandemien abgeleitet aus den Erfahrungen von LEOSS.

diese Methodik große Untersuchungen mit geringem Personalaufwand. Gleichzeitig bestehen Limitationen in der Übertragbarkeit von bspw. elektronischen Verordnungen in tatsächlichen Gebrauch oder von ICD-10 kodierten Diagnosen in Komorbiditäten. Auch im Rahmen der Fachdiskussionen um Real World Evidenz in der Nutzenbewertung von Arzneimitteln wurden von Seiten des Instituts für Qualität und Wirtschaftlichkeit im Gesundheitswesen (IQWiG) Limitationen von Krankenkassendaten oder Daten aus elektronischen Patient:innenakten wie bspw. das Fehlen wesentlicher Störgrößen genannt [34].

Verschiedene Institutionen wie die Europäische Arzneimittelbehöre (EMA), das europäische Netzwerk für die Bewertung von Gesundheitstechnologien (EUnetHTA) oder das Deutsche Netzwerk für Versorgungsforschung (DNVF) haben in diesem Kontext Stellungnahmen zur Eignung von Registern in der Nutzenbewertung erarbeitet und Qualitätsindikatoren definiert [35-39]. Unter Einbezug dieser Vorgaben (z. B. Registerprotokoll, Entscheidungsgremien, Kodierhandbuch, Änderungsverzeichnis, Terminologie, automatisierte Qualitätsprüfungen) kann LEOSS als hochwertiges System zur Datenerhebung gelten. Mit Blick auf die FAIR-Prinzipien wurde ein besonderer Fokus auf Wiederverwendbarkeit und Nachnutzbarkeit gelegt [10]: Daten [40] wie auch Metadaten [16] sind unter eindeutiger Identifikation auffindbar (Findability) und kontinuierlich abrufbar (Accessibility). Im MDM-Portal liegen alle Datenpunkte kodiert mit UMLS (Unified Medical Language System) vor (Interoperability), wie oben ausgeführt wurden Daten zu weiteren europäischen und internationalen Datensammlungen beigetragen (Reuse).Vorteilhaft erwies sich die dynamische und iterative Anpassung des Datensatzes in Zusammenarbeit mit Fachexpert:innen: Hierdurch konnten zahlreiche fachspezifische und elektronisch nicht oder schlecht verfügbare Datenpunkte definiert und durch den manuellen Dokumentationsprozess auswertbar gemacht werden, was wichtige Beiträge z. B. zu kardiovaskulär vorerkrankten Patient:innen [23], zur Neurologie [24] oder Transplantationsmedizin [25] erlaubte.

Eine weitere Stärke der nicht-interventionellen, anonymen Registerstruktur war die rapide Verbreitungsmöglichkeit. Innerhalb von nur 14 Tagen lagen positive Ethikvoten von 37 Standorten vor. Zum Vergleich finden beim weitaus komplexeren, interventionellen, pseudonymen Nationalen Pandemie Kohorten Netz (NAPKON, https://napkon.de) sechs Monate nach Einreichung immer noch Verhandlungen mit einzelnen Ethikkommissionen statt. Großbritannien zeigt allerdings, dass auch komplexe interventionelle Arzneimittelstudien nicht notwendigerweise spät starten müssen: Die dort initiierte Studie The Randomised Evaluation of COVID-19 therapy (RECOVERY, https://www.recoverytrial.net/) zur Evaluation 13 verschiedener Therapieansätze begann etwa zeitgleich mit LEOSS mit der Rekrutierung und zählte bereits nach 15 Tagen fast 1000 Einschlüsse an 132 Zentren [41,42]. 
Erkenntnisse, die Leitlinien beeinflussen, konnten während der ersten Welle in Deutschland nicht generiert werden. Gründe hierfür waren vornehmlich mangelnde Ressourcen. Insgesamt zeigte sich, dass die zeitlichen Vorteile durch den einfachen und finanziell günstigen Rollout des Registers gegenüber einer randomisierten klinischen Studie zumindest teilweise durch die Notwendigkeit der aufwendigen Datennachbereitung und der sorgfältigen Berücksichtigung von Störgrößen wieder aufgehoben wurden.

Insgesamt konnte mit LEOSS gut demonstriert werden, wie sich in einer Pandemiesituation in kürzester Zeit eine umfangreiche klinische Datensammlung international implementieren lässt. Schlüsselelemente waren die enge Zusammenarbeit mit Ethikkommissionen und Datenschutzbeauftragten in der Anfangsphase und der iterative Prozess der Erweiterung und Verbesserung des Datensatzes auch während der Rekrutierung ( $\triangleright \mathbf{A b b}$. 4). Eine ungelöste Frage bleibt, wie in der gebotenen Eile dennoch rasch personelle Ressourcen für ein Projekt gewonnen und dann auch kurzfristig förderrechtlich korrekt eingesetzt werden können, um nach der Startphase auch den Aufwand von Datentransfer und -analyse zu bewältigen. Deutlich wurde auch das Potenzial einer zentralen Hierarchisierung wichtiger Studienkomponenten: Austausch elektronisch verfügbarer Routinedaten, vertiefte Dokumentation der nicht elektronisch verfügbaren Daten, prospektive Rekrutierung für Begleituntersuchungen über die Routine hinaus und prospektive, interventionelle klinische Studien. Hierfür wurden mit NAPKON, in das viele Erfahrungen aus LEOSS eingeflossen sind, und der COVID-19 Data Exchange Platform (CODEX) im Netzwerk Universitätsmedizin (NUM, https://netzwerk-universitaetsmedizin.de/) wichtige Grundsteine gelegt.

\section{Danksagung}

Wir sprechen allen Beteiligten in LEOSS unseren großen Dank für Ihr Engagement aus, insbesondere dem DZIF und der Willy Robert Pitzer Foundation, sowie A. Menke, A. Schmitt, A. Brinker, C. Brünn und M. Brechtel aus der Arbeitsgruppe, die LEOSS ebenfalls unterstützen. Ein besonderer Dank geht an die aktiven Studienzentren (https://leoss.net/enrolled-sites-ethic-votes/), stellvertretend hier die Mitglieder des Board of Investigators: Bundeswehr Zentralkrankenhaus Koblenz (D. Rauschning); Elblandklinikum Riesa (J. Schubert); Elisabeth-Krankenhaus Essen (I. Voigt); Evangelisches Stadtkrankenhaus Saarbrücken (M. Neufang); Hacettepe University (M. Akova); Johannes Wesling Klinikum Minden (K. Wille); Katholisches Klinikum Bochum (K. Hellwig); Kliniken Maria Hilf Mönchengladbach (J. vom Dahl); Klinikum Braunschweig (J. Kielstein); Klinikum Bremen-Mitte (C. Piepel); Klinikum Dortmund (M. Hower); Klinikum Ernst von Bergmann (L. Tometten); Klinikum Ingolstadt (S. Borgmann); Klinikum Leverkusen (L. Eberwein); Klinikum Passau (J. Lanznaster); Krankenhaus St. Joseph-Stift Dresden (L. Walter); Kreuznacher Diakonie Hunsrück (W. Rimili); Marienhospital Herne (T. Westhoff); Medizinische Hochschule Hannover (G. Beutel); Praxis am Ebertplatz Köln (C. Wyen); Richmond Research Institute (J. Taubel); Robert-Bosch-Krankenhaus Stuttgart (K. Rothfuss); Städtisches Klinikum Karlsruhe (C. Degenhardt); Technische Universität München (C. Spinner); Technische Universität München, Kinderklinik (U. Behrends); Universitätsklinikum Augsburg (C. Römmele); Universitätsklinikum Bonn (J. Nattermann); Universitätsklinikum Carl
Gustav Carus Dresden (K. de With); Universitätsklinikum des Saarlandes, Homburg (R. Bals); Universitätsklinikum Düsseldorf (B. Jensen); Universitätsklinikum Erlangen (R. Strauss); Universitätsklinikum Essen (S. Dolff); Universitätsklinikum Frankfurt (M. Vehreschild); Universitätsklinikum Freiburg (S. Rieg); Universitätsklinikum Giessen und Marburg (J. Trauth); Universitätsklinikum Heidelberg (U. Merle); Universitätsklinikum Jena (M. Ruethrich); Universitätsklinikum Köln (N. Jung); Universitätsklinikum München, LMU (M. von Bergwelt-Baildon); Universitätsklinikum Regensburg (F. Hanses); Universitätsklinikum Schleswig-Holstein, Kiel (A. Friedrichs); Universitätsklinikum Schleswig-Holstein, Lübeck (J. Rupp); Universitätsklinikum Tübingen (S. Göpel); Universitätsklinikum Ulm (B. Grüner); Universitätsklinikum Würzburg (N. Isberner); ZIBP Berlin (S. Grunwald).

\section{Finanzierung}

LEOSS erhielt finanzielle Unterstützung durch das Deutsche Zentrum für Infektionsforschung (DZIF) und die Willy Robert Pitzer Stiftung.

Interessenkonflikt

Die Autorinnen/Autoren geben an, dass kein Interessenkonflikt besteht.

Literatur

[1] WHO. COVID-19 Public Health Emergency of International Concern (PHEIC) global research and innovation forum: towards a research roadmap. COVID-19 Public Health Emergency of International Concern (PHEIC) global research and innovation forum: towards a research roadmap 2020; 2020

[2] Bayerisches Landesamt für Gesundheit und Lebensmittelsicherheit RK-I. Beschreibung des bisherigen Ausbruchsgeschehens mit dem neuartigen Coronavirus SARS-CoV-2 in Deutschland (Stand: 12. Februar 2020). Epidemiologisches Bulletin 2020; 7: 3-4. doi:10.25646/647

[3] WHO. WHO Coronavirus Disease (COVID-19) Dashboard In 2021

[4] Stausberg J, Maier B, Bestehorn K et al. Memorandum Registry for Health Services Research: Update 2019. Gesundheitswesen 2020; 82: e39-e66. doi:10.1055/a-1083-6417

[5] Lew TW, Kwek TK, Tai D et al. Acute respiratory distress syndrome in critically ill patients with severe acute respiratory syndrome. JAMA 2003; 290: 374-380. doi:10.1001/jama.290.3.374

[6] Fowler RA, Webb SA, Rowan KM et al. Early observational research and registries during the 2009-2010 influenza A pandemic. Crit Care Med 2010; 38: e120-e132. doi:10.1097/CCM.0b013e3181d20c77

[7] Tsang TK, Wu P, Lin Y et al. Effect of changing case definitions for COVID-19 on the epidemic curve and transmission parameters in mainland China: a modelling study. Lancet Public Health 2020; 5 : e289-e296. doi:10.1016/S2468-2667(20)30089-X

[8] Tang N, Li D, Wang X et al. Abnormal coagulation parameters are associated with poor prognosis in patients with novel coronavirus pneumonia. J Thromb Haemost 2020; 18: 844-847. doi:10.1111/ jth. 14768 
[9] Hoffmann W, Latza U, Baumeister SE et al. Guidelines and recommendations for ensuring Good Epidemiological Practice (GEP): a guideline developed by the German Society for Epidemiology. Eur J Epidemiol 2019; 34: 301-317. doi:10.1007/s10654-019-00500-x

[10] Wilkinson MD, Dumontier M, Aalbersberg IJ et al. The FAIR Guiding Principles for scientific data management and stewardship. Sci Data 2016; 3: 160018. doi:10.1038/sdata.2016.18

[11] GO FAIR International Support and Coordination Office (GFISCO). GO FAIR. Im Internet: https://www.go-fair.org/fair-principles/; Stand: 23.02.2021

[12] Der Bundesbeauftragte für den Datenschutz und die Informationsfreiheit. Positionspapier zur Anonymisierung unter der DSGVO unter besonderer Berücksichtigung der TK-Branche (29.06.2020). Im Internet: https://www.bfdi.bund.de/DE/Infothek/Transparenz/ Konsultationsverfahren/01_Konsulation-Anonymisierung-TK/ Positionspapier-Anonymisierung.pdf; Stand: 23.02.2021

[13] Malin B, Loukides $G$, Benitez $\mathrm{K}$ et al. Identifiability in biobanks: models, measures, and mitigation strategies. Hum Genet 2011; 130: 383-392. doi:10.1007/s00439-011-1042-5

[14] Jakob CEM, Kohlmayer F, Meurers T et al. Design and evaluation of a data anonymization pipeline to promote Open Science on COVID-19. Sci Data 2020; 7: 435. doi:10.1038/s41597-020-00773-y

[15] Dugas M, Neuhaus P, Meidt A et al. Portal of medical data models: information infrastructure for medical research and healthcare. Database (Oxford) 2016; pii: bav121. doi:10.1093/database/bav121

[16] LEOSS study group. Lean European Open Survey on SARS-CoV-2 Infected Patients. In medical data models (mdm) portal 2021. doi: $10.21961 / \mathrm{mdm}: 42476$

[17] Deutsche Gesellschaft für Infektiologie e.V. (dgi). LEOSS - Europäisches Fallregister für Patientinnen und Patienten mit SARS-CoV-2Infektion. (2020). Im Internet: https://www.dgi-net.de/leoss-europaeisches-fallregister-fuer-patientinnen-und-patienten-mit-sars-cov-2-infektion/; Stand: 23.02.2021

[18] Deutsche Gesellschaft für Kardiologie - Herz- und Kreislaufforschung e.V. (DGK). Europäisches LEOSS-Register zu SARS-CoV-2-Infektionen eingerichtet - Bitte um Teilnahme. (07.04.2020). Im Internet: https:// dgk.org/news/europaeisches-leoss-register-zu-sars-cov-2-infektioneneingerichtet-bitte-um-teilnahme/; Stand: 23.02.2021

[19] Deutsche Gesellschaft für Neurologie (DGN). LEOSS-Register erfasst MS-spezifische Kriterien bei SARS CoV II-infizierten Patienten: Bitte pflegen Sie Ihre Patientendaten ein!. (25.03.2020). Im Internet: https://dgn.org/neuronews/neuronews/leoss-register-erfasst-msspezifische-kriterien-bei-sars-cov-ii-infizierten-patienten-bitte-pflegen-sie-ihre-patientendaten-ein/; Stand: 23.02.2021

[20] Deutsches Konsortium für Translationale Krebsforschung (DKTK). Gemeinsames DKTK-Patientenregister. (16.04.2020). Im Internet: https://dktk.dkfz.de/ueber-uns/news/gemeinsames-dktk-patientenregister; Stand: 23.02.2021

[21] Deutsches Zentrum für Herz-Kreislauf-Forschung e.V. (DZHK). Europaweites Register für Coronavirus-Patienten. (02.04.2020). Im Internet: https://dzhk.de/aktuelles/news/artikel/europaweites-register-fuer-coronavirus-patienten/; Stand: 23.02.2021

[22] Deutsches Zentrum für Infektionsforschung (DZIF). COVID-19: Europäisches Fallregister für Coronavirus-Patienten im Aufbau. (19.03.2020). Im Internet: https://www.dzif.de/de/covid-19-europaeisches-fallregister-fuer-coronavirus-patienten-im-aufbau; Stand: 23.02.2021

[23] Cremer S, Jakob C, Berkowitsch A et al. Elevated markers of thromboinflammatory activation predict outcome in patients with cardiovascular comorbidities and COVID-19 disease: insights from the LEOSS registry. Clin Res Cardiol 2020; 110: 1029-1040. doi:10.1007| s00392-020-01769-9
[24] Huber MK, Raichle C, Lingor P et al. Outcomes of SARS-CoV-2 Infections in Patients With Neurodegenerative Diseases in the LEOSS Cohort. Mov Disord 2021; 36(4): 791-793. doi:10.1002/mds.28554

[25] Hugo C, Stecher M, Dolff $S$ et al. Solid organ transplantation is not a risk factor for COVID-19 disease outcome. Transplant International 2021; 34: 378-381. doi:10.1111/tri.13795

[26] Hugo C, Strassburg C, Stecher M et al. Stable and safe organ procurement and transplantation during SARS-CoV-2 pandemic in Germany. Transplant International 2020; 33: 1335-1336. doi:10.1111/ tri.13704

[27] Jakob CEM, Borgmann S, Duygu F et al. First results of the "Lean European Open Survey on SARS-CoV-2-Infected Patients (LEOSS)". Infection 2021; 49: 63-73. doi:10.1007/s15010-020-01499-0

[28] Pilgram L, Eberwein L, Wille $K$ et al. Clinical course and predictive risk factors for fatal outcome of SARS-CoV-2 infection in patients with chronic kidney disease. Infection 2021; 49: 725-737. doi:10.1007/ s15010-021-01597-7

[29] Ruthrich MM, Giessen-Jung C, Borgmann S et al. COVID-19 in cancer patients: clinical characteristics and outcome-an analysis of the LEOSS registry. Ann Hematol 2021; 100: 383-393. doi:10.1007/s00277-02004328-4

[30] Schoser B, Baum P, Boentert M et al. SARS-CoV-2/COVID-19 und neuromuskuläre Erkrankungen. DGNeurologie 2020; 3: 310-320. doi:10.1007/s42451-020-00198-2

[31] Infectious Diseases Data Observatory. COVID-19 clinical data platform. https://iddo.cognitive.city/cognitive/apps/cognitive-cityhome?page = iddo-public. Stand: 14.04.2021

[32] Wong AY, MacKenna B, Morton CE et al. Use of non-steroidal anti-inflammatory drugs and risk of death from COVID-19: an OpenSAFELY cohort analysis based on two cohorts. Ann Rheum Dis 2021; 80: 943-951. doi:10.1136/annrheumdis-2020-219517

[33] Yang JM, Koh HY, Moon SY et al. Allergic disorders and susceptibility to and severity of COVID-19: A nationwide cohort study. J Allergy Clin Immunol 2020; 146: 790-798. doi:10.1016/j.jaci.2020.08.008

[34] Institut für Qualität und Wirtschaftlichkeit im Gesundheitswesen (IQWiG). Rapid Report A19-43 Version 1.1: Versorgungsnahe Daten zum Zwecke der Nutzenbewertung. In: 2020

[35] Harkener S, Stausberg J, Hagel C et al. Towards a Core Set of Indicators for Data Quality of Registries. Stud Health Technol Inform 2019; 267: 39-45. doi:10.3233/SHTI190803

[36] Europäisches Netzwerk für Health Technology Assessment (EUnetHTA). Vision paper on the sustainable availability of the proposed Registry Evaluation and Quality Standards Tool (REQueST) 2019

[37] Institut für angewandte Versorgungsforschung. Anwendungsbegleitende Daten in der Nutzenbewertung: Empfehlungen zur Evidenzgenerierung und -auswertung 2019

[38] Stausberg J. Stand der Register in Deutschland im Überblick. Ärztezeitung 2020; Heft 10

[39] Lange S. Evidenzhierarchie und Registerdaten: Die Perspektive des IQWiG Ärztezeitung 2020; Heft 10

[40] Jakob C, Kohlmayer F, Meurers T et al. LEOSS Public Use File. In: Zenodo 2020. doi:10.5281/zenodo.4059716

[41] RECOVERY. First patients enrolled in new clinical trial of possible COVID-19 treatments. In: 2020

[42] RECOVERY. RECOVERY Trial rolled out across the UK. In: 2020 